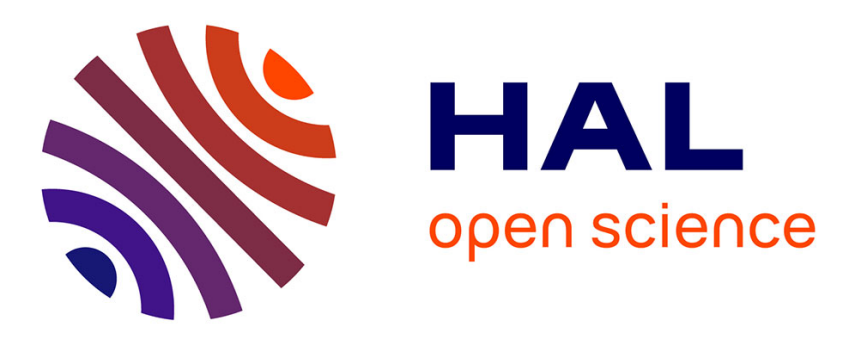

\title{
Limites de la prise en compte de la diversité des publics scolaires en Guyane.
}

\author{
Sophie Alby, Jeannine Ho-A-Sim
}

\section{To cite this version:}

Sophie Alby, Jeannine Ho-A-Sim. Limites de la prise en compte de la diversité des publics scolaires en Guyane.. Raisons Educatives, 2010, Numéro thématique "Globalisation et transformations des systèmes éducatifs : enjeux, réalités et av, pp.169-190. halshs-00665368

\section{HAL Id: halshs-00665368 \\ https://shs.hal.science/halshs-00665368}

Submitted on 2 Feb 2012

HAL is a multi-disciplinary open access archive for the deposit and dissemination of scientific research documents, whether they are published or not. The documents may come from teaching and research institutions in France or abroad, or from public or private research centers.
L'archive ouverte pluridisciplinaire HAL, est destinée au dépôt et à la diffusion de documents scientifiques de niveau recherche, publiés ou non, émanant des établissements d'enseignement et de recherche français ou étrangers, des laboratoires publics ou privés. 


\section{Référence :}

Alby, Sophie \& Ho-A-Sim, Jeannine. 2010. Limites de la prise en compte de la diversité des publics scolaires en Guyane. In : A. Akkari \& J-P. Payet (coord.), Raisons Educatives, numéro thématique "Globalisation et transformations des systèmes éducatifs : enjeux, réalités et avatars de la scolarisation dans les pays du Sud», 169-190.

La Guyane présente une situation linguistique et culturelle exceptionnelle, non pas tant en raison du plurilinguisme qui la caractérise (celui-ci est omniprésent dans le monde) mais de la configuration de ce plurilinguisme, notamment en contexte scolaire : allophonie d'élèves étrangers mais aussi d'élèves français, grande diversité linguistique, écart culturel important entre le monde de l'école et celui de l'environnement familial et quotidien. Cette diversité linguistique et culturelle de l'école en Guyane constitue donc un enjeu essentiel pour une région qui, en tant que département français, est difficilement assimilable aux «pays du Sud », mais qui pourtant en présente de nombreuses caractéristiques de part son, histoire coloniale ${ }^{1}$.

Face à cette situation, l'école française centralisatrice se doit donc d'envisager une transformation de son système éducatif, afin de mieux l'adapter au public qu'elle accueille. Cette transformation peut porter sur différents niveaux: programmes, formation des enseignants, etc. Toutefois elle se heurte à la conception du système éducatif par des décideurs, qui s'appuient sur une réalité fantasmée, une vision holistique de l'éducation en France. De surcroît, l'appartenance de la France au contexte européen suppose une prise en compte des directives élaborées à ce niveau.

Aussi, la Guyane est-elle face à un enjeu majeur : celui d'une nécessaire réinterprétation des contenus, des méthodes d'enseignement, et même des principes qui gouvernent cet enseignement. Ses acteurs ne peuvent toutefois agir qu'en se basant sur les textes officiels qui régissent le système éducatif.

Comment dans un tel contexte, et face à de tels enjeux, résoudre le paradoxe que constitue une école française dans un département d'Outre-Mer, situé en Amérique du Sud ? C'est à cette question que nous tenterons de répondre en décrivant, dans un premier temps, les caractéristiques de ce contexte éducatif, l'inadaptation des décisions de l'état en matière éducative. Cette description s'appuiera sur des analyses de documents, et sera exemplifiée par

\footnotetext{
${ }^{1}$ Voir Puren (2007) pour une présentation de l'histoire des politiques linguistiques éducatives en Guyane, dans le contexte colonial et postcolonial.
} 
des études de cas issus de différents espaces éducatifs de la région. Nous chercherons ensuite à déterminer quels sont les éléments d'adaptation essentiels, en émettant des propositions en termes de contenus d'enseignement, de profil des enseignants et de formation de ces derniers.

1. L'école face à l'enjeu de la diversité

L'école en Guyane s'appuie sur les programmes élaborés au plan national. Elle applique donc à l'ensemble de son public ce qui est valable pour tout enfant scolarisé en France, quelle que soit sa langue ou sa culture d'origine, au nom du principe d'égalité. Si en soit ce principe est fondé, on peut cependant s'interroger sur son adéquation avec le principe d'équité. Tous les élèves de France sont donc égaux (égalité) quant aux enseignements qu'on leur dispense, mais le fait-on réellement dans le respect absolu de ce qui est dû à chacun (équité) ? Sur le plan linguistique, l'école en Guyane pratique effectivement une inégalité de traitement des élèves, puisque les locuteurs natifs du français sont favorisés par rapport aux autres.

\subsection{La Guyane, un département français}

7077 kilomètres séparent la France métropolitaine de la Guyane. Cette distance n'est pas seulement géographique : elle est aussi linguistique et culturelle. Ce département français d'outre-mer, d'une superficie de 83934 kilomètres, qui fait partie de l'ensemble dit du plateau des Guyanes (Brésil, Guyane française, Surinam, Guyana, Venezuela), est largement recouvert par la forêt amazonienne. Sa population de 229000 habitants (INSEE, 2009) ${ }^{2}$ vit principalement sur le littoral guyanais (41\% de la population vit sur l'île de Cayenne) et, dans une moindre mesure, le long des deux fleuves frontières, le Maroni (frontière avec le Surinam) et l'Oyapock (frontière avec le Brésil).

La population guyanaise est composée de diverses communautés linguistiques et culturelles. A l'heure actuelle, il est parfois difficile de tracer des limites claires entre ces communautés, tant les contacts sont importants entre elles. Aussi, l'appartenance à un groupe ne signifie pas que ses membres parlent la langue de ce groupe et inversement. On compte environ une trentaine de langues pratiquées dans le département. Certaines peuvent être qualifiées de langues de Guyane, au sens où elles présentent les caractéristiques des langues régionales ; elles sont listées dans le rapport Cerquiglini (1999), que nous évoquons ci-dessous :

\footnotetext{
${ }^{2}$ Source $:$ http://www.insee.fr/fr/themes/tableau.asp?reg_id=25\&ref_id=poptc001
} 
- Six des langues amérindiennes appartenant à trois familles linguistiques : la famille tupi-guarani (teko et wayampi), la famille caribe (kali'na et wayana), la famille arawak (lokono et pahikwaki). Ces langues étaient parlées sur le territoire avant la colonisation.

- Des langues créoles à base lexicale anglaise ou langues businenge, le nengee tongo (qui regroupe trois variétés : le ndyuka, l'aluku et le pamaka) et le saamaka. Ces langues s'inscrivent dans l'histoire de l'esclavage et du marronnage ${ }^{3}$.

- Une langue créole à base lexicale française, le créole guyanais. C'est la seule langue a avoir effectivement le statut de langue régionale.

- Une langue asiatique, le hmong (famille des langues hmong-mien), parlée par un groupe originaire du Laos. Ses membres se sont implantés en Guyane en 1977, après avoir fui le Laos et acquis le statut de réfugiés politiques.

En dehors de ces langues, on en trouve d'autres issues d'une immigration plus récente, comme par exemple :

- Des langues européennes parlées dans des pays de la Caraïbe ou de l'Amérique du Sud (espagnol, portugais, anglais, néerlandais).

- Des langues asiatiques (mandarin, cantonais, hakka, lao, vietnamien).

- Des langues créoles à base lexicale française (créole haïtien, créole saint-lucien).

Des langues de France y sont aussi parlées : créole martiniquais, créole guadeloupéen, créole réunionnais. Et bien sûr le français y est la langue de scolarisation.

Une des particularités de ce département est donc bien sa grande diversité qui, du fait de l'implantation géographique des populations, génère des contacts linguistiques et culturels intenses, et une situation plurilingue et pluriculturelle très spécifique.

\subsection{La diversité linguistique et culturelle, un enjeu pour l'école}

La communauté scientifique et les instances internationales (UNESCO) ou européennes (Conseil de l'Europe) qui s'intéressent aux questions éducatives s'accordent aujourd'hui sur la nécessité d'une prise en compte des langues et des cultures locales dans l'enseignement: «toute personne a droit à une éducation et à une formation de qualité qui respectent

\footnotetext{
${ }^{3}$ Les esclaves « marrons » sont ceux qui se sont enfuis des plantations.
} 
pleinement son identité culturelle » (UNESCO, 2001, article 5). Les Etats doivent de ce fait s'engager à « susciter, à travers l'éducation, une prise de conscience de la valeur positive de la diversité culturelle et améliorer à cet effet tant la formulation des programmes scolaires que la formation » ainsi qu'à «encourager la diversité linguistique - dans le respect de la langue maternelle - à tous les niveaux de l'éducation, partout où c'est possible, et [à] stimuler l'apprentissage du plurilinguisme dès le plus jeune âge » (UNESCO, 2001, points 6 et $7 \mathrm{du}$ Plan d'action joint à la déclaration).

L'UNESCO incite donc les états non seulement à reconnaître la diversité culturelle et linguistique, mais également à en tenir compte dans l'élaboration de ses programmes, ou dans la formation des enseignants. Parallèlement, le Conseil de l'Europe (2000) insiste sur la nécessité de s'appuyer sur les compétences linguistiques et culturelles des élèves pour construire les apprentissages, dans le but de les conduire à une compétence plurilingue et pluriculturelle. On trouve donc dans ces documents des idées fondamentales, qui ont des implications autant d'ordre didactique que politique : la nécessité de reconnaitre la diversité linguistique et culturelle, de prendre appui sur celle-ci pour élaborer les apprentissages, mais aussi d'amener les élèves à développer leurs compétences dans ce domaine. L'école ne peut donc faire l'impasse sur une prise en compte des langues et des cultures des élèves en Guyane, pour diverses raisons : psychologiques : «favoriser le développement personnel et la réussite scolaire de l'enfant de langue maternelle autre que le français »; culturelles : «participer aux côté des familles à la sauvegarde du patrimoine linguistique et culturel »; politiques : «favoriser la reconnaissance de la diversité de fait des sociétés et partant la compréhension entre les groupes » (Salaün, 2009 : 32).

Cette dernière raison renvoie à un enjeu plus large : le fait que «le dialogue interculturel constitue le meilleur gage pour la paix »(UNESCO, 2001). Aussi, pour permettre aux élèves de mieux vivre ensemble, la prise en compte de leur diversité est un point de départ incontournable. En effet, l'un des objectifs de l'école primaire «est d'apprendre à l'enfant à reconnaître ce qui le distingue des autres et à se faire reconnaître comme personne », condition pour qu'il parvienne ensuite à vivre avec les autres (ministère de l'éducation nationale, 2008). De même, pour accéder à la maîtrise du langage, des savoirs scolaires, ou plus généralement à la culture de l'école, il est essentiel de s'appuyer sur la représentation que les élèves se font de l'objet enseigné (Astolfi, 1992), donc de partir de ce qui est déjà-là : leurs compétences linguistiques et culturelles. 


\subsection{Complexité des situations scolaires dans le département}

Ceci implique donc de s'interroger, avant toute proposition en termes de politique éducative, sur la particularité de la situation concernée. Or, en Guyane comme ailleurs, il ne s'agit pas seulement d'évoquer un public scolaire ou une situation scolaire, mais bien des publics dans toute leur diversité et des situations scolaires, qui varient fortement d'une école - voire même d'une classe - à une autre, que ce soit au niveau linguistique ou au niveau culturel.

La situation linguistique dans les écoles en Guyane ne peut s'envisager globalement. Pour pouvoir en mesurer toute la complexité, il convient de s'intéresser - par le biais d'approches microsociolinguistiques - à des situations particulières. C'est ainsi que les travaux sociolinguistiques menés dans le département ces 10 dernières années (Alby, 2005 ; Alby \& Léglise, 2005, 2007a ; Léglise, 2004, 2005, 2007 ; Léglise \& Migge, 2005, ; Léglise \& Puren, 2005) ont permis de mettre en évidence la pluralité des situations scolaires, sans toutefois pouvoir les appréhender toutes. Cette pluralité se décline à différents niveaux : rapports des élèves au français, langue(s) parlée(s) par les élèves, langue(s) parlée(s) dans l'environnement des élèves, etc. Ainsi, une partie d'entre eux sont (mono, bi ou plurilingues) avant leur scolarisation, locuteurs d'une ou de plusieurs langues autres que le français ; d'autres ont eu un contact, plus ou moins intense, avec la langue de l'école, avant leur entrée dans le système scolaire ; enfin certains sont bi ou plurilingues (avec le français). Par ailleurs, l'allophonie des élèves n'est pas uniquement le fait de l'immigration, puisqu'une partie d'entre eux sont Français tout en ayant une langue maternelle autre que le français ; de même, un enfant de migrants peut très bien compter le français parmi ses langues premières. De ce point de vue, les profils linguistiques des classes sont très variés, allant d'une classe où tous les élèves parlent la langue de l'école à une classe où aucun d'entre eux ne la parle. On peut ainsi distinguer trois types de situations scolaires au regard des profils sociolinguistiques des élèves :

- Type 1: des classes présentant une grande hétérogénéité des langues parlées dans l'environnement familial, et une «homogénéité » dans la maîtrise de la langue de l'école.

- Type 2: des classes plus homogènes au niveau des langues parlées dans la famille, avec des degrés de maîtrise assez variés de la langue de l'école.

- Type 3: des classes plus homogènes du point de vue des langues parlées, avec une majorité d'élèves qui ne maîtrisent pas du tout ou très peu la langue de scolarisation.

Le tableau 1 exemplifie ces trois types : 


\begin{tabular}{|c|c|c|}
\hline \multicolumn{3}{|c|}{ Tableau 1 : exemples de la diversité scolaire (comparaison de trois classes) ${ }^{4}$} \\
\hline $\begin{array}{c}\text { Jean Macé (Cayenne) } \\
\text { Classe de CM1 } \\
(2006-2007)\end{array}$ & $\begin{array}{c}\text { Rolland Lucille (Kourou) } \\
\text { Classe de CP } \\
(2006-2007)\end{array}$ & $\begin{array}{l}\text { Moutende (Apatou) } \\
\text { Classe de CP } \\
(2005-2006)\end{array}$ \\
\hline $\begin{array}{l}\text { Tous les élèves ont le } \\
\text { français dans leur répertoire } \\
\text { et en ont une assez bonne } \\
\text { maîtrise ; mais en plus de } \\
\text { cette langue, } 12 \text { autres sont } \\
\text { parlées dans l'environnement } \\
\text { familial : créole guyanais, } \\
\text { portugais, anglais, espagnol, } \\
\text { créole haïtien, saamaka, } \\
\text { néerlandais, aluku, tamoul, } \\
\text { ourdou, mandarin, cantonais. }\end{array}$ & $\begin{array}{c}17 \text { élèves sont locuteurs de } \\
\text { saamaka avec des degrés de } \\
\text { maîtrise de la langue } \\
\text { française assez variés, } 1 \\
\text { élève ne parle que le français, } \\
\text { les autres parlent le } \\
\text { portugais, l'espagnol, le } \\
\text { créole guyanais, le } \\
\text { néerlandais. }\end{array}$ & $\begin{array}{c}\text { Sur } 26 \text { élèves, } 23 \text { ne parlent } \\
\text { absolument pas le français } \\
\text { les } 3 \text { autres l'ont appris à } \\
\text { l'école maternelle. Ils sont } \\
\text { tous locuteurs du nenge } \\
\text { (langue qui regroupe } 3 \\
\text { variétés proches } \\
\text { linguistiquement : aluku, } \\
\text { ndyuka, pamaka). }\end{array}$ \\
\hline
\end{tabular}

Ce tableau met en évidence la diversité des situations scolaires d'un point de vue linguistique, sans pour autant réduire les compositions possibles de ces classes à des types, car il existe encore bien d'autres profils. Il convient en outre d'observer que les langues parlées par les élèves sont elles-mêmes très diversifiées, et comportent des degrés d'éloignement plus ou moins marqués avec la langue de l'école: langues amérindiennes, langues créoles à base lexicale française, langues créoles à base lexicale anglaise, langues européennes, langues d'Asie, etc. Or, bien que la distance linguistique joue un rôle non négligeable dans l'acquisition de la langue française, elle n'est que rarement prise en compte tant dans les pratiques que dans les discours officiels.

Cette distance linguistique trouve son écho dans la distance culturelle. Les élèves sont ainsi confrontés à de multiples références culturelles qu'ils doivent articuler pour pouvoir se construire dans l'environnement scolaire (Tap, 1991). Une comparaison de quatre groupes d'élèves (Ho-A-Sim, 2004) montre ainsi que d'un groupe à l'autre les référents culturels et le rapport à l'école se construisent de manières très différentes. Ces groupes sont identifiés sur une base communautaire, avec un groupe d'élèves kali'na de la commune d'Awala-Yalimapo, et trois groupes d'élèves de la commune de Mana : Businenge, Hmong et Créoles guyanais. Les deux communes sont localisées au nord-ouest de la Guyane et sont assez proches l'une de l'autre.

Les enfants kali'na vivent selon un mode semi-traditionnel, sur un site que leur communauté occupe depuis des décennies. Ils sont locuteurs du kali'na, mais sont aussi familiers de la

\footnotetext{
${ }^{4}$ Le tableau s'appuie sur des travaux de recherche menés de 2006 à 2009 dans le cadre de l’ERTé n³2 de l'IUFM de la Guyane (Alby, 2009).
} 
langue française qui est pour eux, le plus souvent, une langue seconde. Leurs parents ont été scolarisés dans leur jeunesse dans les homes catholiques ${ }^{5}$. Malgré la violence de cette expérience, ils transmettent à leurs enfants l'importance de l'instruction scolaire en assurant un suivi scolaire.

Les enfants businenge, de la communauté ndjuka, sont issus de l'immigration consécutive à la guerre civile au Surinam voisin. La situation socio-économique de leurs familles est plus précaire, et le français n'est que rarement parlé dans leur environnement. La plupart ne fréquentent pas l'école maternelle, et doivent intégrer l'école primaire en tant que primoarrivants. Leurs parents, quant à eux, ont été rarement scolarisés. Dans cette communauté, l'autonomie des enfants est développée très tôt : ils sont capables assez rapidement de se prendre en charge ainsi que leurs jeunes frères et sœurs. Il semble que les parents attendent la même autonomie au niveau des apprentissages scolaires, et les plus âgés n'aident pas particulièrement les plus jeunes lors des devoirs à domicile. Les aînés sont d'ailleurs souvent en situation d'échec scolaire; une fois rentrés à la maison, ils s'occupent à des tâches domestiques ou liées à des activités de subsistance. Le discours sur l'école est globalement positif (Ho-A-Sim, 2004), mais il ne se traduit pas en actes favorisant l'approfondissement des apprentissages scolaires. Les enfants sont livrés à eux-mêmes la plupart du temps.

Les enfants hmong sont dans une situation socio-économique plutôt favorable. Leurs parents sont maraîchers, et les enfants doivent aider aux travaux des champs après la classe. Ces enfants sont Français, mais la langue de scolarisation est loin d'être pratiquée hors de l'école. Dans le village et à l'école, les élèves ne parlent le français que lorsqu'ils s'adressent aux professeurs. Le village est enclavé, ce qui le rend peu propice à des échanges en français. Aussi, en dehors de l'école, ces enfants ne sont en contact avec la langue française que lors des jours de vente au marché. Les parents ont eux-mêmes été scolarisés au moins jusqu'au collège. Mais peu d'entre eux ont entrepris des études supérieures. Au moment où l'enquête a été menée (1999-2000), il n'y avait pas de bacheliers dans le village. Il faut peut-être en attribuer la raison à la célébration précoce (vers 15 ou 16 ans) de mariages traditionnels qui ont pour conséquence l'entrée dans la vie d'adulte : travail dans l'agriculture et naissance des enfants. Mais le site de Javouhey où se trouve cette communauté est aujourd'hui saturé, et les parents ont pris conscience de l'importance de la réussite scolaire des jeunes pour prétendre à d'autres débouchés. Cependant, comme pour les enfants ndjuka, cette prise de conscience ne se traduit pas véritablement en actes par des conduites d'accompagnement scolaire. Les

\footnotetext{
${ }^{5}$ Internats tenus par des autorités religieuses.
} 
enfants sont sur ce plan relativement livrés à eux-mêmes, et doivent développer une autonomie précoce.

Enfin, les enfants de la communauté créole grandissent souvent dans un environnement bilingue français-créole guyanais, mais ont du mal à s'engager dans leur scolarité. Ils habitent pour la plupart le bourg de Mana, et leurs parents ont des situations sociales relativement stables.

La présentation de ces trois groupes montre des profils culturels et langagiers très variés, comportant des écarts avec la culture de l'école plus ou moins grands. Or l'école, qui a pour rôle de contribuer à la construction des individus, ne suscite pas suffisamment d'échos en eux pour qu'ils s'y investissent; de plus, les perspectives qui s'offrent à eux ne sont souvent pas bien comprises, ou ne correspondent pas aux attentes véhiculées dans la communauté d'appartenance. On peut, à juste titre, s'interroger sur la transmission d'un modèle qui veut que la réussite scolaire soit une condition de la réussite sociale.

2. L'échec de l'école face à la diversité

\subsection{L'échec scolaire en Guyane}

On ne peut s'empêcher en comparant ces situations de pluralités linguistiques et culturelles avec la politique centralisatrice de l'Etat en matière de programme scolaire, de s'intéresser aux résultats de l'école française en Guyane. Or, les chiffres mettent clairement en évidence un échec de l'école dans sa politique actuelle. En effet, les résultats de l'année 2008 dans les domaines considérés comme fondamentaux à l'école primaire (le français et les mathématiques), pour les classes de $\mathrm{CE} 1$ et de $\mathrm{CM} 2$, sont très largement inférieurs à ceux obtenus pour l'ensemble de France, comme le montrent les graphiques suivants ${ }^{6}$ :

Graphiques 1 à 4 : évaluations de mai 2009 en CE1 et de janvier 2009 en CM2

\footnotetext{
${ }^{6}$ Source : Ministère de l'Education nationale : résultats des évaluations des acquis des élèves de l'école primaire - académie de la Guyane. http://www.education.gouv.fr/evaluation_des_acquis/acad28/guyane.html
} 

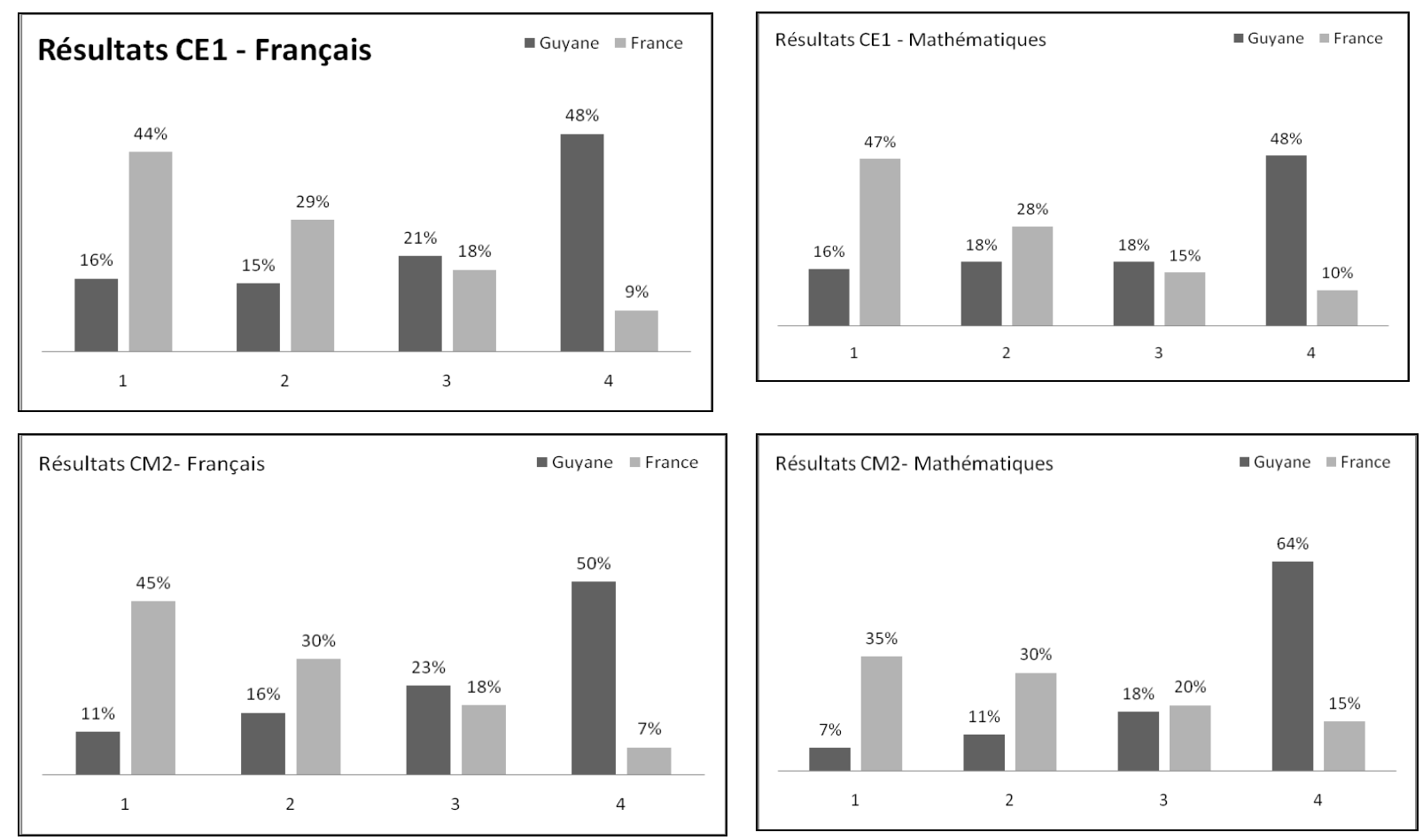

Légende : 1 : plus de 39 bonnes réponses, les acquis sont très solides. 2 : entre 30 et 39 réponses, les acquis sont bons et seront développés dans les mois à venir. 3 : entre 20 et 29 bonnes réponses, les acquis sont encore fragiles, ils seront à consolider dans les mois à venir. 4 : les élèves ont moins de 20 bonnes réponses, les acquis ne sont pas suffisants, les élèves doivent bénéficier d'une aide spécifique.

Ces chiffres, comparés à la moyenne nationale, mettent en évidence un échec scolaire important dans les matières considérées comme essentielles à l'école primaire en France. Plus précisément, les élèves dont les acquis sont insuffisants et qui doivent bénéficier d'une aide spécifique (situation 4) sont majoritaires :

- En CE1, en français et en mathématiques, on compte 48\% d'élèves dans ce cas (contre $9 \%$ pour la moyenne nationale en français et $10 \%$ pour les mathématiques) ;

- En CM2, ce sont 50\% des élèves qui sont dans ce cas en français (contre 7\% pour la moyenne nationale) et $64 \%$ en mathématiques (contre $15 \%$ pour la moyenne nationale).

Si l'on met ces chiffres en regard avec d'autres indicateurs de l'échec scolaire dans le département, on ne peut que conclure à l'échec du système scolaire en Guyane et de la politique centralisatrice de l'Etat français en matière d'éducation. En effet, les chiffres des sources officielles mettent l'académie de la Guyane en queue de peloton de toutes les académies de France ${ }^{7}$ pour de nombreux critères comme le montre le tableau $2^{8}$ :

\footnotetext{
${ }^{7}$ Hexagone et DOM-TOM : 30 académies.
} 
Tableau 2 : classement de la Guyane parmi les académies de France en 2002-2003

\begin{tabular}{|l|l|l|l|l|}
\hline Critère & $\begin{array}{l}\text { Première } \\
\text { ou dernière } \\
\text { académie }\end{array}$ & Se lit & $\begin{array}{l}\text { Positionnement } \\
\text { de la Guyane }\end{array}$ & $\begin{array}{l}\text { Moyenne } \\
\text { nationale }\end{array}$ \\
\hline $\begin{array}{l}\text { Retards } \\
\text { scolaires en 6e }\end{array}$ & $\begin{array}{l}\text { La Réunion } \\
(25 \%)\end{array}$ & $\begin{array}{l}\text { Académie comportant le } \\
\text { taux le plus important de } \\
\text { retard scolaire en 6 }\end{array}$ & $29^{\mathrm{e}}(5,8 \%)$ & $4 \%$ \\
\hline $\begin{array}{l}\text { proportion de } \\
\text { collégiens en } \\
\text { ZEP/REP }\end{array}$ & $\begin{array}{l}\text { Corse } \\
(58,8 \%)\end{array}$ & $\begin{array}{l}\text { Académie comportant le } \\
\text { plus important de } \\
\text { collégiens en ZEP/REP }\end{array}$ & $29^{\mathrm{e}}(45,8 \%)$ & $22,73 \%$ \\
\hline $\begin{array}{l}\text { Sortie sans } \\
\text { qualification }\end{array}$ & $\begin{array}{l}\text { Guyane } \\
(28 \%)\end{array}$ & $\begin{array}{l}\text { Académie comportant le } \\
\text { taux le plus important } \\
\text { d'élèves sortis sans } \\
\text { qualification du système } \\
\text { scolaire }\end{array}$ & $30^{\mathrm{e}}(28 \%)$ & $9 \%$ \\
\hline $\begin{array}{l}\text { Passage de 3 } \\
\text { en } \\
\text { générale ou } \\
\text { techonologique }\end{array}$ & $\begin{array}{l}\text { Guyane } \\
(40,6 \%)\end{array}$ & $\begin{array}{l}\text { Académie comportant le } \\
\text { taux le moins important } \\
\text { d'élèves passant d'une } 3^{\mathrm{e}} \\
\text { à une 2 } \\
\text { technologique gérale ou }\end{array}$ & $30^{\mathrm{e}}(40,6 \%)$ & $57,66 \%$ \\
\hline $\begin{array}{l}\text { Proportion de } \\
\text { bacheliers }\end{array}$ & $\begin{array}{l}\text { Guyane } \\
(32,2 \%)\end{array}$ & $\begin{array}{l}\text { Académie comportant le } \\
\text { taux le moins important } \\
\text { de bacheliers }\end{array}$ & $30^{\mathrm{e}}(32,2 \%)$ & $60,61 \%$ \\
\hline
\end{tabular}

La Guyane est dans trois cas sur cinq la dernière de toutes les académies de France, et pour les deux autres cas, elle est en avant-dernière position.

Les écarts culturels et linguistiques des élèves face à la culture et à la langue de l'école ne suffisent certes pas à eux seuls à expliquer ces résultats. Cependant, on peut poser l'hypothèse qu'ils jouent un rôle non négligeable, et que leur prise en considération pourrait contribuer à lutter contre l'échec scolaire. Or, si à l'heure actuelle on compte quelques actions dans ce domaine, elles sont relativement récentes et peu développées dans certains cas. En outre, il convient d'observer que cet écart culturel et linguistique se trouve renforcé par l'extranéité du corps enseignant vis-à-vis du public scolaire (Alby, 2009 ; Ho-A-Sim \& Alby, 2006).

2.2. Les actions contre l'échec scolaire

Comme le rappellent Migge \& Renault-Lescure (2009), la scolarisation des enfants de Guyane s'est développée progressivement «depuis les débuts de la colonisation au XVIIe

\footnotetext{
${ }^{8}$ Chiffres de 2002-2003, publiés par la Direction de l'évaluation et de la prospective du Ministère de l'éducation nationale.
} 
siècle, et de façon différenciée suivant les populations » (p.56). Ces auteurs précisent qu'elle a d'abord touché les enfants de colons, puis à partir de 1848 (date de l'abolition de l'esclavage) et de 1888 (laïcisation), elle a commencé à concerner les enfants des anciens esclaves. Durant une assez longue période, elle ne s'est adressée qu'à une partie de la population guyanaise, essentiellement celle qui se situait dans les milieux urbains du littoral. Il faut donc attendre la fin de la première moitié du $20^{\mathrm{e}}$ siècle, avec la départementalisation (1946), pour que les autres groupes guyanais, notamment les Businenge et les Amérindiens, soient scolarisés. Ils le seront, dans un premier temps, dans le cadre des «homes » catholiques, et ce n'est qu'au cours des années 1970 qu'ils commencent à intégrer les écoles publiques.

Dans tous les cas, ce qui caractérise cette scolarisation, c'est qu'elle est fondée sur le principe d'assimilation et de francisation. Ainsi, comme le souligne Puren (2007), l'adaptation au contexte guyanais est «quasi-nulle: les écoles de la colonie ne se distinguent de leurs homologues métropolitaines ni par les programmes, ni par les horaires, ni par la langue d'instruction »(p.282). En ce qui concerne les programmes et les horaires, cette situation perdure encore aujourd'hui. Il existe cependant quelques efforts d'adaptation récents pour prendre en considération les langues et les cultures locales. Mais ceux-ci sont souvent le fait d'initiatives extérieures au système éducatif ${ }^{9}$, et prennent souvent des formes expérimentales qui ont du mal à se développer ${ }^{10}$.

On distingue, à l'heure actuelle, deux formes d'adaptation au contexte guyanais à l'école primaire. La première, les dispositifs CLIN-CRI ${ }^{11}$, est une action qui existe depuis les années 1970 au niveau national, et prend en compte le fait qu'une partie des élèves scolarisés dans les écoles françaises n'ont pas le français pour langue maternelle. Mais, selon les textes, elle ne s'intéresse qu'à des élèves de nationalité étrangère (en Guyane, des élèves de nationalité française y sont malgré tout parfois accueillis), ne tient pas compte de leurs particularités linguistiques et culturelles, et propose un modèle pédagogique visant à compenser la non maîtrise de la langue française, considérée comme un manque, une lacune chez les élèves. Les dispositifs CLIN-CRI ont pour fonctions de «faciliter l'adaptation [des] jeunes au système français d'éducation en développant des aides adaptées à leur arrivée » et «assurer dès que

\footnotetext{
${ }^{9}$ Lorsqu'elles sont internes, elles sont souvent individuelles et ont des difficultés à se faire reconnaître par l'institution.

${ }^{10}$ Pour une récapitulation de l'histoire de la prise en compte des langues et des cultures des élèves à l'école, voir Alby et Léglise, 2007b et Puren, 2007.

${ }^{11}$ Classes d'initiation et Cours de rattrapage intégré.
} 
possible l'intégration dans le cursus scolaire ordinaire» (circulaire du Ministère de l'éducation nationale $\mathrm{n}^{\circ} 2002-100$ du 25 avril 2002).

Les élèves sont « regroupés - tous cycles confondus - pour un enseignement prodigué selon la méthodologie du français langue seconde [ou de scolarisation] » (alby \& tabournel, sous presse), dans le but de les amener à la maîtrise du français langue de scolarisation. On ne peut donc pas parler, dans ce cas, d'une réelle alternative locale, les enseignements dispensés étant globalement les mêmes que dans les autres CLIN-CRI de France. On peut toutefois observer que le CASNAV ${ }^{12}$ de la Guyane qui est chargé du suivi et de la formation de ce dispositif, fait depuis une dizaine d'années de nombreux efforts pour contextualiser ces enseignements en proposant, entre autres, des méthodes de langage (A toi la parole, Langage en fête) et de lecture (Tiki), dont les référents culturels sont proches des élèves. Les auteurs de Langage en fête précisent ainsi, sur le site de l'académie de la Guyane ${ }^{13}$, que ce manuel est conçu pour « les élèves de Guyane non francophones », et qu'elle permet à ceux-ci de «s'identifier aux personnages mis en scène », car «toutes les communautés présentes en Guyane apparaissent au fil des leçons ». Le manuel Tiki, qui se présente comme une suite logique de Langage en fête, reprend ce principe en y ajoutant un élément linguistique : «l'originalité réside dans le fait que le choix des phonèmes, adapté au public pluri-ethnique de la Guyane, a été établi d'après les études réalisées par l'Institut de Recherche et de Développement sur la comparaison entre les phonèmes du français et ceux des langues présentes en Guyane ». Ces ouvrages ciblent toutefois l'ensemble du public non francophone, sans prendre en compte les spécificités des différentes situations scolaires. Il serait en effet peu aisé d'éditer des manuels adaptés à chaque situation.

La seconde forme d'adaptation au contexte guyanais, concerne des dispositifs nationaux ou locaux (et expérimentaux), qui s'appuient sur la particularité linguistique d'une partie du public scolaire. Ils sont au nombre de trois: deux qui sont reconnus au niveau national, l'enseignement des langues et cultures régionales et le dispositif d'enseignement renforcé des langues régionales; et un qui se fait au niveau local à titre expérimental, le dispositif «Intervenants en langues maternelles ».

L'enseignement des langues vivantes régionales (LVR) s'inscrit dans un cadre réglementaire qui a connu plusieurs étapes historiques. La loi n51-46 du 11 janvier 1951 relative à l'enseignement des langues et des dialectes locaux (aujourd'hui abrogée), dite loi Deixonne,

\footnotetext{
${ }^{12}$ Centre académique pour la scolarisation des nouveaux arrivants et des enfants du voyage.

${ }^{13}$ http://www.ac-guyane.fr/article297.html
} 
avait pour objectif tout à la fois de défendre la langue française et de protéger les langues régionales. Elle constitue la première reconnaissance officielle du droit à l'existence des langues régionales, et est une loi à la fois linguistique et scolaire. Si cette loi a été abrogée, les langues régionales continuent néanmoins de pouvoir être enseignées à l'école, une heure et demie au minimum au minimum et trois heures au maximum. Dans les programmes de 2008, elles apparaissent désormais dans l'encart concernant l'enseignement des langues vivantes, et des programmes sont en cours de réalisation pour chaque langue régionale. En Guyane, la seule langue ayant le statut de langue régionale de France est le créole (créole guyanais). Elle est enseignée comme langue vivante régionale dans un peu plus de 300 classes, et pratiquement toutes les circonscriptions comptent un conseiller pédagogique en LVR (le créole guyanais peut être une option au baccalauréat, et il existe un CAPES créole).

Le dispositif d'enseignement renforcé des langues régionales s'appuie sur le décret 2001-733 du 31 juillet 2001. Il peut être implanté dans les académies disposant d'un conseil académique des langues régionales. Il a permis la création de quelques classes bilingues français-créole guyanais depuis deux ans. C'est sous l'influence des courants de pensée antillais que le créole guyanais a été introduit dans les écoles dès 1986, et ce, sous l'impulsion d'une inspectrice de l'Education nationale, Mme Sonia Francius (Alby \& Léglise, 2007b).

Certes il y a avec ces deux dispositifs, la prise en compte d'une langue et d'une culture du département guyanais ; mais du fait qu'ils sont issus d'une approche nationale de la question, des problèmes se posent d'un point de vue didactique. En effet, l'enseignement des LVR touche des publics qui peuvent être locuteurs natifs, peu locuteurs ou non locuteurs. Des directives et un programme communs pour des publics aussi différents semblent donc inappropriés. Il convient de ce fait d'engager une réflexion didactique de fond sur les modalités d'enseignement de cette langue dans le département.

Le dispositif «Intervenants en langues maternelles » s'appuie sur la notion de langue régionale. En effet, dans un rapport datant de 1999, Cerquiglini liste, parmi les langues de Guyane pouvant accéder au statut de langue régionale en dehors du créole guyanais, six des langues amérindiennes qui y sont parlées, deux langues businenge et le hmong. C'est en se basant sur ce rapport que des linguistes du Centre d'Etudes des Langues Indigènes d'Amérique (CELIA) ont proposé au Rectorat de la Guyane, à la fin des années 1990, la création d'une structure d'enseignement de ces langues qui s'intitulait initialement le dispositif «Médiateurs culturels et bilingues». L'objectif était d'accueillir les enfants à l'école primaire dans leur langue première, de leur prodiguer des enseignements dans cette 
langue, l'hypothèse étant que des élèves scolarisés dans leur propre langue auraient plus de chance de s'intégrer dans le système scolaire, d'accéder aux savoirs scolaires, mais aussi de se structurer en tant que bilingues, tant dans leur langue première que dans la langue de l'école. Ce dispositif existe encore aujourd'hui, mais il a connu de nombreux aléas, liés à des raisons institutionnelles pour la plupart. L'idée d'alphabétiser les élèves dans leur langue première a été rejetée par l'institution, les intervenants en langues maternelles se voyant peu à peu cantonnés au rôle d'accueil des élèves dans les premières années de leur scolarisation. Cet aspect est certes essentiel, mais très éloigné des principes de départ du projet. De plus, la pérennisation du dispositif est rendue peu aisée par l'absence de véritable statut pour les intervenants qui, ne bénéficiant pas du statut d'enseignant, ont des contrats à durée déterminée (contrats emplois jeunes, contrats aidés) qui ne peuvent excéder un certain nombre de renouvellements. Passé ce stade, ils sont licenciés, car l'éducation nationale ne veut pas être obligée de les embaucher avec des contrats à durée indéterminée.

Des tentatives existent donc en Guyane pour prendre en compte les spécificités locales, mais elles ont bien souvent du mal à se développer ou à perdurer dans de bonnes conditions. De plus, elles se focalisent généralement sur un public très spécifique, ne permettent pas de tenir compte de la pluralité des situations et n'influent pas sur les contenus des programmes qui restent nationaux. Les raisons de cette inadaptation sont en grande partie liées au poids de l'idéologie monolingue française héritée de la révolution, qui conduit au «divorce entre plurilinguisme et école » dénoncé par Coste (1991, p.170).

\subsection{Des alternatives politico-juridiques à la question éducative}

La Guyane française, en tant que département d'Outre-Mer est soumise, sur le plan éducatif, à la politique nationale. Pourtant son statut et l'évolution de celui-ci pourrait permettre des changements dans ce domaine.

La France d'outre-mer n'est pas un ensemble homogène du point de vue des statuts de ses territoires respectifs. On regroupe sous cette appellation les départements et régions d'outremer (DOM-ROM), les collectivités d'outre-mer et les collectivités sui generis. En tant que département et région d'outre-mer, la Guyane a le même statut que les départements et régions de France métropolitaine, et est considérée comme région ultra-périphérique sur le plan européen. Mais en tant que DOM-ROM, elle dépend de l'article 73 (Legifrance, 2003) 
qui stipule que les lois et règlements «peuvent faire l'objet d'adaptation tenant aux caractéristiques et contraintes particulières de ces collectivités ». Cependant, l'éducation n'est pas directement concernée par cette loi. L'article 74 (Legifrance 2003) permettrait une meilleure adaptation à ce niveau, comme c'est le cas en Nouvelle-Calédonie où les programmes de l'école primaire sont pensés localement (donc adaptés au contexte) et différenciés des programmes nationaux. En effet, la loi organique a permis de donner à la Nouvelle-Calédonie la compétence de gérer elle-même l'enseignement primaire et ses programmes, qui peuvent être adaptés aux réalités culturelles et linguistiques. Il en va de même pour la formation des maîtres ${ }^{14}$. Cependant, les habitants de la Guyane ont, le 10 janvier 2010, voté contre le passage à l'article 74 confirmant leur souhait de rester sous le régime de l'article 73 . Les seules options possibles pour une adaptation du système éducatif sont donc toujours le recours à des lois d'habilitation permettant des modifications temporaires ${ }^{15}$ de son fonctionnement.

A l'heure actuelle, les seuls aménagements possibles relèvent de la loi n84-747 du 2 août 1984, qui mentionne, dans ses articles 21 et 23, les adaptations concernant les langues régionales. L'article 21 évoque la question de leur enseignement :

Le Conseil régional détermine, après avis du comité de la culture, de l'éducation et de l'environnement, les activités éducatives et culturelles complémentaires relatives à la connaissance des langues et des cultures régionales, qui peuvent être organisées dans les établissements scolaires relevant de la compétence de la région. Ces activités, qui peuvent se dérouler pendant les heures d'ouverture des établissements concernés, sont facultatives et ne peuvent se substituer ni porter atteinte aux programmes d'enseignement et de formation définis par l'État. [...].

L'article 23 autorise quant à lui des aménagements sur le plan culturel :

Les régions de Guadeloupe, de Guyane, de Martinique et de la Réunion définissent les actions qu'elles entendent mener en matière culturelle, après avis ou, le cas échéant, sur proposition des collectivités territoriales et du comité de la culture, de l'éducation et de l'environnement. À cette fin, le Conseil régional élabore un programme culturel régional, notamment dans le domaine des langues régionales, de la littérature, des arts plastiques, musicaux et cinématographiques.

De plus, certaines des langues non reconnues comme langues régionales peuvent bénéficier d'une forme de protection, du fait de l'article 33 de la loi d'orientation pour l'outre-mer (loi 2000-1207 du 13 décembre 2000) : «l'État et les collectivités locales encouragent le respect,

\footnotetext{
${ }^{14}$ Avis n ${ }^{\circ} 364747$ du 10 octobre 2000. Section de l'intérieur. Outremer.

${ }^{15}$ Les lois d'habilitation ne sont valables que pour la durée du mandat de l'assemblée qui les a votées.
} 
la protection et le maintien des connaissances, innovations et pratiques des communautés autochtones et locales fondées sur leurs modes de vie traditionnels $[\ldots] »$, ce qui peut permettre d'arguer de la nécessité de leur sauvegarde en les introduisant à l'école. L'article 34 de cette même loi évoque aussi la nécessité de la protection des langues régionales.

Ainsi, si le cadre législatif actuel ne permet pas véritablement de mettre en œuvre des programmes adaptés au plan local, certains textes permettent des aménagements. Néanmoins, l'évolution institutionnelle semble être la seule condition d'un véritable changement et d'une réelle adaptation au contexte. Dans cette expectative, il convient de penser aux conditions d'élaboration des programmes. Ces derniers devront à la fois tenir compte des réalités locales, et faciliter l'accès à un savoir scolaire permettant de poursuivre des études au sein du système éducatif français, que ce soit à l'antenne locale de l'Université des Antilles et de la Guyane (Institut d'enseignement supérieur de la Guyane), ou dans des filières offertes uniquement dans d'autres universités françaises. De plus, la prise en considération de ces réalités locales doit se baser sur une analyse fine, rationnelle, du contexte scolaire, et tenir compte de la diversité des situations. C'est en ce sens que nous posons que la pluralité doit être au centre de tout débat sur l'élaboration d'une alternative locale.

3. La prise en compte de la pluralité, une alternative locale

La diversité, qu'elle soit linguistique, culturelle, socio-économique, ou autre, doit être pensée comme constitutive de l'école. Elle s'impose donc logiquement à cette institution en tant que constat, mais plus encore en tant que point de départ et objectif de toute son organisation. L'alternative locale à la situation éducative en Guyane pourrait donc être, d'une part, de s'appuyer sur la pluralité pour construire les apprentissages scolaires tous domaines de l'école confondus, en incluant les modes éducatifs propres aux communautés concernées, et d'autre part, de viser la construction, chez les élèves, d'une compétence à vivre dans une société plurielle. Il s'agit donc de développer chez ces derniers des attitudes positives vis-à-vis de leurs propres langues et cultures (estime de soi) pour favoriser leur ouverture à l'autre, à l'inconnu. Si l'on se pose, à la suite d'Abdallah-Pretceille et Porcher (1996), la question fondamentale de savoir quel individu l'école peut-elle, veut-elle, doit elle former dans le contexte guyanais, la réponse doit être pensée au niveau local, dans une perspective décentralisée. Or, nous considérons que l'école en Guyane doit former les individus dont elle 
a la charge à une compétence plurielle (plurilingue et pluriculturelle) tout autant qu'à l'acquisition des compétences scolaires fondamentales. Cette compétence plurielle comprend des éléments qui touchent autant à la culture qu'à la langue et peut se définir comme

\begin{abstract}
la compétence à communiquer langagièrement et à interagir culturellement possédée par un locuteur qui maîtrise, à des degrés divers, plusieurs langues et a, à des degrés divers, l'expérience de plusieurs cultures, tout en étant à même de gérer l'ensemble de ce capital langagier et culturel. L'option majeure est de considérer qu'il n'y a pas là superposition ou juxtaposition de compétences toujours distinctes, mais existence d'une compétence plurielle, complexe, voire composite et hétérogène, qui inclut des compétences singulières, voire partielles, mais est une en tant que répertoire disponible pour l'acteur social concerné. (Coste, Moore \& Zarate, 1997, p.12)
\end{abstract}

Un tel point de vue peut être central dans l'élaboration de programmes spécifiques à la Guyane, incluant les savoirs des communautés sur les objets enseignés à l'école, que ce soit dans le domaine de la découverte du monde (savoirs traditionnels sur la diversité biologique par exemple), ou dans le domaine des mathématiques (ethno-mathématiques, par exemple).

Mais au-delà des contenus, il peut aussi donner lieu à la mise en place d'approches didactiques spécifiques s'appuyant sur l'idée même de la pluralité, notamment les approches plurielles qui incluent - entre autres - l'éveil aux langues et au langage ou l'approche interculturelle. Des expériences de ce type sont déjà en cours en Guyane, notamment en éveil aux langues et au langage. Elles ont lieu dans une zone géographiquement délimitée pour faciliter les déplacements du chercheur lors du suivi de l'expérimentation : l'île de Cayenne et Kourou. Quatre circonscriptions de l'Académie (Cayenne nord, Kourou, Matoury et Cayenne sud) sont concernées avec cinq classes expérimentales en 2007/2008 et 2008/2009, et onze en 2009/2010. Les activités s'adressent à des élèves de cycle 2 et 3, et le public présente des caractéristiques assez variées, que ce soit sur le plan socio-économique, linguistique (langues premières et maîtrise de la langue de scolarisation) ou culturel (écart plus ou moins grand avec la culture de l'école). A titre d'exemple, le graphique 5 met en évidence la diversité des langues parlées par les élèves : 
Graphique 5 : diversité des langues parlées par les élèves de cycles 2 et 3 à Cayenne nord, Kourou, Matoury et Cayenne sud

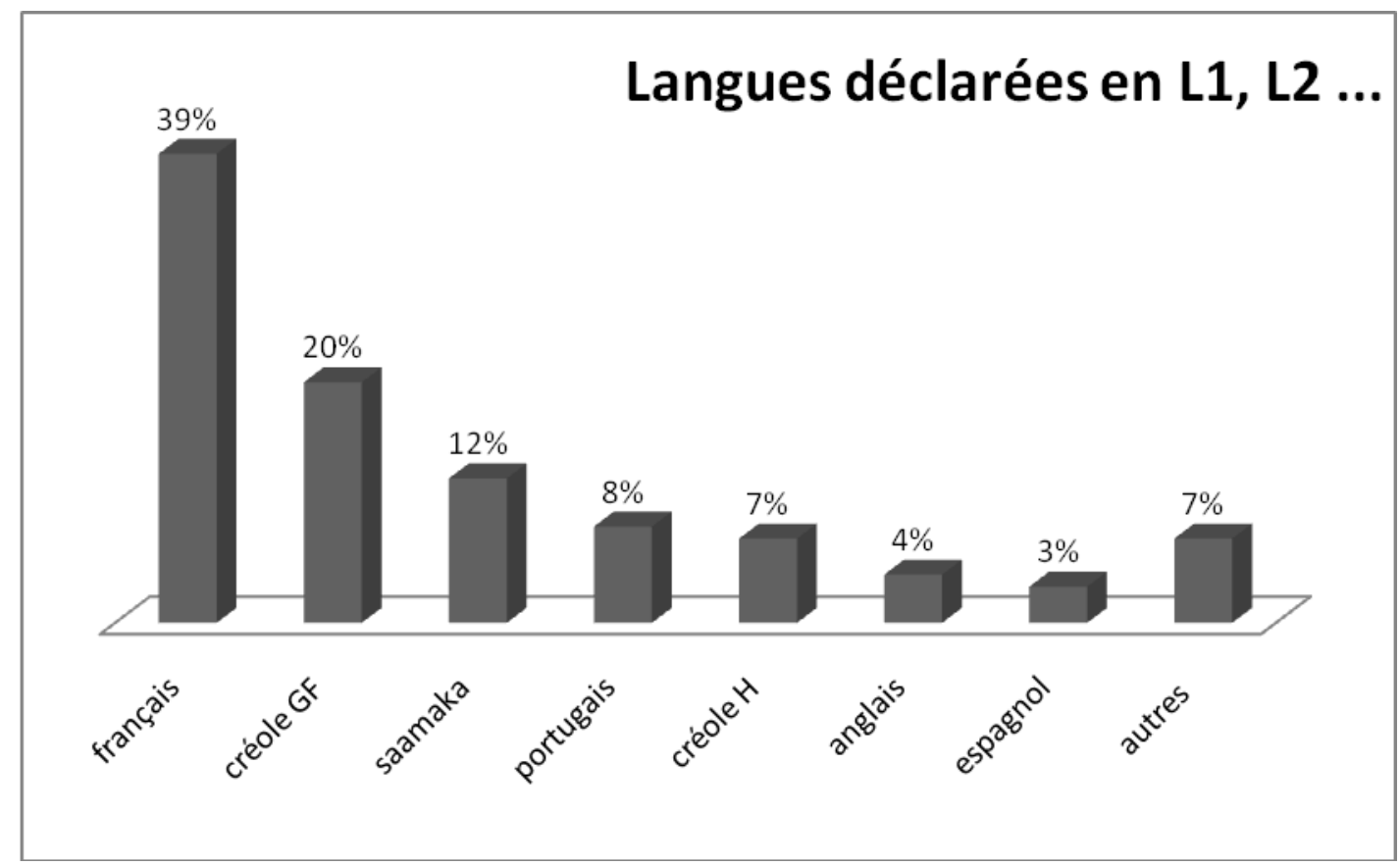

Ces données ont été recueillies au travers de divers types d'enquêtes sociolinguistiques menées dans le cadre des travaux de recherche de l'ERTé n³2 de l'IUFM de la Guyane (Alby, 2009): données en situation (pratiques déclarées lors des séances filmées), par entretiens (réponses orales pour les élèves de cycle 2, et questionnaire écrit pour les élèves de cycle 3). Les chiffres s'appuient sur les déclarations de 272 élèves qui ont déclaré en tout 492 langues, avec une moyenne de 1,8 langue par élève. L'importance du français est la plus grande dans la mesure où il apparaît souvent dans les cas de bi-plurilinguisme. La catégorie «autres » comprend des langues dont le pourcentage est inférieur à 3\%. Les classes concernées présentent donc une forte diversité linguistique, et toutes les langues appartenant au répertoire des élèves ont été prises en compte. Le principe consiste à partir des compétences acquises des élèves et à valoriser toutes leurs langues sans qu'aucune hiérarchie ne soit établie entre elles. Il s'agissait, pour les expérimentateurs, de construire au travers de ces séances les conditions pour que se développe une compétence plurilingue telle qu'elle a été définie ci-dessus, en se focalisant plus spécifiquement sur deux aspects : apprendre à apprendre les langues et apprendre à vivre ensemble. S'il est peu aisé, à ce stade de l'expérimentation, de se prononcer sur le premier objectif, les évaluations qualitatives menées ont permis de montrer que le second a été le plus souvent atteint (Bitard, 2008). La prise en considération des langues et des cultures de tous les élèves et leur découverte par les autres 
ont permis de développer des attitudes positives, non seulement envers ces langues et ces cultures, mais aussi à l'égard de ceux qui les pratiquent.

Toutefois, les expérimentations menées - et plus globalement les approches plurielles - ne se limitent pas à la question des langues. Comme le souligne Byram (2009), il convient aussi d'engager une réflexion sur la pluralité dans tous les domaines de l'école primaire. C'est ainsi que certains enseignants travaillent à la mise en place de projets plaçant l'interculturalité au cœur des enseignements des disciplines non linguistiques : la numération en mathématiques, les systèmes d'écriture en graphisme, les instruments de musique du monde, le fait historique considéré selon des points de vue différents (à partir de manuels scolaires de pays voisins comme le Brésil et le Surinam). Cette réflexion sur la diversité permet de revenir aux savoirs scolaires. Ainsi, un enseignant de cycle 3, lors d'un travail sur la division, a proposé à ses élèves issus de l'immigration de montrer à leurs camarades leurs manières de construire cette opération mathématiques. La comparaison d'autres modes opératoires, la confrontation à une manière de faire inconnue, a permis aux élèves de prendre plus facilement du recul et à considérer non plus la division comme une tâche scolaire supplémentaire, mais de l'appréhender en tant que concept. Il en va de même pour des activités d'étude de la langue. Les élèves ont bien souvent du mal à envisager la langue de scolarisation comme un objet d'étude : ils la conçoivent plus aisément comme un outil de communication qui ne prête pas à questionnement. La confrontation à d'autres langues, la comparaison de mécanismes linguistiquespermet aux élèves de prendre conscience des universaux du langage et de son fonctionnement, en se défocalisant des aspects sémantiques. Aussi la pluralité n'est-elle pas un obstacle à l'acquisition des savoirs scolaires. Elle permet au contraire de mieux y accéder.

\section{En conclusion : le global au service du local}

L'approche de l'éducation que nous proposons dans cet article n'est locale qu'au sens de l'adaptation des contenus et des méthodes d'enseignement au contexte. Mais le principe qui lui est sous-jacent, la pluralité, peut être envisagé comme gouvernant toute situation éducative. Or, c'est bien ce qui est prôné dans les différents documents émanant du Conseil de l'Europe. Il s'agit en effet de faire de l'éducation plurilingue et interculturelle un projet, pour reprendre le titre de documents élaborés pour la Division des politiques linguistiques de ce dernier par Cavalli, Coste, Crisan et Van de Ven (2009) et par Byram (2009). 
Ce projet d'une éducation plurilingue et interculturelle au cœur même du fonctionnement du système éducatif suppose, en tout premier lieu, de tenir compte des acquis des élèves, donc de leurs langues et de leurs cultures. Ainsi, l'école pourra remplir son rôle en tirant «parti de la diversité des langues [pour son projet éducatif et agir en tant qu'espace institutionnel privilégié pour l'accueil, la valorisation [...] du plurilinguisme déjà là, contribuant ainsi à l'inclusion sociale»(Cavalli \& al., 2009, p.6). De même, elle doit mettre en place des stratégies éducatives visant à sensibiliser à la pluralité culturelle et à favoriser le dialogue interculturel (Byram, 2009). Pour que les élèves apprennent à vivre ensemble (pour reprendre un des domaines de l'école primaire), à entrer dans un dialogue interculturel, ils doivent développer leurs compétences interculturelles pour pouvoir appréhender des situations nouvelles du point de vue d' « autres » (Byram, 2009).

Références bibliographiques

Abdallah-Pretceille, Martine \& Porcher, Louis. (1996). Education et communication interculturelle. Paris : Presses Universitaires de France.

Alby, Sophie. (2005). Une approche bilinguiste du contact des langues : discours bilingues d'enfants kali'na en situation scolaire. Trace, 47, 96-112.

Alby, Sophie. (2009). La formation des enseignants dans le contexte guyanais. In J. Vernaudon \& V. Fillol (éd.), Vers une école plurilingue dans les collectivités françaises d'Océanie et de Guyane (pp.225-250). Paris : L'Harmattan.

Alby, Sophie \& Léglise, Isabelle. (2005). L’enseignement en Guyane et les langues régionales, réflexions sociolinguistiques et didactiques. Marges Linguistiques, 10, 245-261. Alby, Sophie \& Léglise, Isabelle. (2007a). Le paysage sociolinguistique de la Guyane : un état des lieux des recherches. In S. Mam-Lam-Fouck (Ed.), Comprendre la Guyane d'aujourd'hui (pp.469-479). Cayenne : Ibis Rouge Editions. 
Alby, Sophie \& Léglise, Isabelle. (2007b). La place des langues des élèves à l'école en contexte guyanais : quatre décennies de discours scientifiques. In Serge Mam-Lam-Fouck (Coord.), Comprendre la Guyane d'aujourd'hui (pp.439-452). Cayenne : Ibis Rouge Editions.

Alby, Sophie \& Tabournel, Patricia. (sous presse). Le dispositif des CLIN-CRI en Guyane. In Rodica Ailincai \& Marie-Françoise Crouzier (Coords.), A l'école de la Guyane : pratiques éducatives pour des compétences plurilingues et pluriculturelles. Cayenne : CRDP Guyane.

Bitard, Gilles. (2008). La valorisation des langues des élèves à l'école en Guyane. L'éveil aux langues au service du développement d'attitudes positives en classe. Mémoire de Master 2 sous la direction de Pierre Dumont, soutenu en octobre 2008 à 1'Université des Antilles et de la Guyane.

Byram, Michael. (2009). Sociétés multiculturelles et individus pluriculturels. Le projet de l'éducation interculturelle. Division des politiques linguistiques, Conseil de l'Europe.

Cavalli, Marisa, Coste Daniel, Crisan, Alexandru \& Van de Ven Piet-Hein. (2009). L'éducation plurilingue et interculturelle comme projet. Division des politiques linguistiques, Conseil de l'Europe.

Cerquiglini, Bernard. (1999). Les langues de France. Rapport au ministre de l'éducation nationale, de la recherche et de la technologie et à la ministre de la culture et de la communication. Avril 1999. http://www.dglflf.culture.gouv.fr/lang$\underline{\text { reg/rapport_cerquiglini/langues-france.html }}$

Conseil de l'Europe. (2000). Cadre européen commun de référence pour les langues. Didier.

Coste, Daniel, Moore, Danièle \& Zarate, Geneviève. (1997). Compétence plurilingue et pluriculturelle. Conseil de l'Europe. 
Ho-A-Sim, Jeannine. (2004). Rapport à la culture, estime de soi et insertion scolaire : le cas des enfants kali'na, ndyuka, hmong et créoles de l'Ouest guyanais. Thèse de doctorat, Université de Toulouse 2.

nir. Formation et pratiques d'enseignement en question, 4, 55-76.Ho-A-Sim, Jeannine \& Alby, Sophie. (2006). Regard sur la formation des enseignants à l'IUFM de la Guyane : quelle adaptation au contexte guyanais ? Des propositions pour l'av

Légifrance. (1996). Loi n̊84-747 du 2 août 1984 relative aux compétences des régions de Guadeloupe, de Guyane, de Martinique et de la Réunion. Version consolidée au 24 février 1996.http://www.legifrance.gouv.fr/telecharger_rtf.do?idTexte=LEGITEXT000006068863\& $\underline{\text { dateTexte }=19960224}$

Légifrance. (2000). Loi n²000-1207 du 13 décembre 2000 d'orientation pour l'outremer. NOR:INTX0000028L. Version consolidée du 7 août 2009. http://www.legifrance.gouv.fr/telecharger_rtf.do?idTexte=LEGITEXT000005630251\&dateTe $\underline{\mathrm{xte}=20090807}$

Legifrance. (2003). Constitution du 4 octobre 1958. Titre XII : des collectivités territoriales. Article 74. Modifié par la loi 2003-276 2003-03-28 art.10 JORF 29 mars 2003. Modifiée par la loi constitutionnelle $n^{\circ} 2003-276$ du 28 mars 2003 - art.10. http://www.legifrance.gouv.fr/affichCodeArticle.do?cidTexte=LEGITEXT000006071194\&id $\underline{\text { Article }=\text { LEGIARTI000006527584\&dateTexte }=\& \text { categorieLien }=\text { cid }}$

Léglise, Isabelle. (2004). Langues frontalières et langues d'immigration en Guyane française. Glottopol, 4, 108-124.

Léglise, Isabelle. (2005). Contacts de créoles à Mana (Guyane française) : répertoires, pratiques, attitudes et gestion du plurilinguisme. Etudes Créoles, 28, 23-57.

Léglise, Isabelle. (2007). Des langues, des domaines, des régions. Pratiques, variations, attitudes linguistiques en Guyane. In Isabelle Léglise \& Bettina Migge (Eds.), 
Pratiques et représentations linguistiques en Guyane : regards croisés (pp.13-30). Paris : IRD Editions.

Léglise, Isabelle \& Migge, Bettina. (Eds). (2007). Pratiques et représentations linguistiques en Guyane : regards croisés. Paris : IRD Editions.

Léglise, Isabelle \& Migge, Bettina. (2005). Contacts de langues issus de mobilités dans un espace plurilingue : approches croisées à Saint-Laurent du Maroni (Guyane). In Cécile Van den Avenne (Ed.), Mobilités et contacts de langues (pp.75-94). Paris : L'Harmattan.

Léglise, Isabelle \& Puren, Laurent. (2005). Usages et représentations linguistiques en milieu scolaire guyanais. In Frédéric Tupin (Ed.), Ecole et éducation, Univers Créoles 5 (pp.67-90). Paris : Anthropos.

Migge, Bettina \& Renault-Lescure, Odile. (2009). Langues et école en Guyane. In Jacques Vernaudon \& Véronique Fillol (Coords.), Vers une école plurilingue dans les collectivités françaises d'Océanie et de Guyane (pp.49-67). Paris : L’Harmattan.

Ministère de l'Education Nationale. (2009). Résultats des évaluations des acquis des élèves de l'école primaire - Académie de la Guyane. http://www.education.gouv.fr/cid1180/direction-evaluation-prospective-performance.html

Ministère de 1’Outre-Mer. (2000). Avis n³64 747 du 10 octobre 2000. http://www.conseil-etat.fr/cde/media/document//avis/364747.pdf

Puren, Laurent. (2007). Contribution à une histoire des politiques linguistiques éducatives mises en œuvre en Guyane française depuis le XIXe siècle. In Isabelle Léglise \& Bettina Migge (Coord.), Pratiques et représentations linguistiques en Guyane : regards croisés (pp.278-295). Paris : IRD Editions.

Salaün, Marie. (2009). Langues locales et école en contexte français : une perspective sociohistorique. In Jacques Vernaudon \& Véronique Fillol (Coords.), Vers une école 
plurilingue dans les collectivités françaises d'Océanie et de Guyane (pp.21-33). Paris :

L'Harmattan.

Tap, Pierre. (1991). Socialisation et construction de l'identité personnelle. In Hanna Malewska-Peyre \& Pierre Tap (Eds.), La socialisation de l'enfance et de l'adolescence (pp.49-75). Paris : PUF.

UNESCO. (2001). Déclaration universelle sur la diversité culturelle. Octobre 2001. 